\title{
A Diffusion-Model-Based Supply-Side Offer Agent
}

\author{
HyungSeon Oh and Robert J. Thomas, Fellow, IEEE
}

\begin{abstract}
Worldwide energy markets have been on a path toward deregulation for several decades. These markets have proven to be difficult to design and run because of their repetitive nature and the externalities provided by reliable grid operation. In a deregulated market environment, our experiments suggest that market participants will pursue their own profit-maximizing objective rather than use an objective that reflects social benefit. Multiagent simulation is useful for gaining insights into market participant behavior under various rules. In this way, market rules can be tested for efficacy and efficiency. In this paper, an agent based on a double-layer diffusion model developed elsewhere [1] is tested and its effectiveness reported.
\end{abstract}

Index Terms-Agent, auction, degree of speculation, diffusion, maximum offer price, nonlinear dynamics, numerical optimization.

\section{INTRODUCTION}

$\mathbf{R}$ ECENT changes in the electric power industry over several decades have led to a less regulated energy market. In all restructured markets, auctions play a major role in determining both the price for electricity as well as generation set points. Market participants make bids and offers, and an independent system operator (ISO) clears the market. We presume that the objective of market participants is to maximize their own profit. Within this framework, a strategic offer strategy must be developed by each participant. Conventional economic modeling approaches have shown a limited ability to explain the strategic behavior of some generating firm, primarily because of the complex cognitive behavior associated with participating in a repeated auction.

Sheble et al. [2]-[4] designed an agent using a genetic algorithm that operates in a simplified market and with zero forecast error. Each agent, including suppliers and consumers, can submit both price and quantity for only one block of energy. The system operator then clears the market, and the agents are paid according to the rules of a discriminatory auction. In such a setup, the offer price and quantity can be assessed relative to its profit, which allows the agent to update future offers. However, the assumptions of one block offers, no forecast error, and a discriminative auction are very different from any real market in operation today.

Manuscript received December 22, 2005; revised May 10, 2006. This work was supported in part by the U.S. Department of Energy through the Consortium for Electric Reliability Technology Solutions (CERTS) and in part by the National Science Foundation Power Systems Engineering Research Center (PSERC). Paper no. TPWRS-00847-2005.

The authors are with the Department of Electrical and Computer Engineering, Cornell University, Ithaca, NY 14850 USA (e-mail: hso1 @ cornell.edu; rjt1@cornell.edu).

Color versions of Figs. 1-4 and 6-10 are available online at http://ieeexplore. ieee.org.

Digital Object Identifier 10.1109/TPWRS.2006.881156
The autonomous agent designed by Bunn et al. [5]-[7] has been applied to a market that assumes a lossless network and no line constraints. Unlike the work of Sheble et al., they allow an agent to offer several blocks. The price and quantity of each block are determined by a linear supply curve defined by two end points that are assigned by reinforcement. The reinforcement algorithm is used to create the parameters that determine offer price and quantity of the blocks. Once all the offers are submitted, a system operator clears the market. The simple nature of reinforcement algorithm allows the agent to work in a nondiscriminatory auction. However, an agent characterized by a linear offer curve may not successfully emulate a real market since the generally observed shape of a supply curve in a uniform price repeated auction is a hockey stick.

In comparison to the work described above, the agent developed by Oh et al. [8] works well in a more realistic market. The technique cannot accommodate a network with line constraints or losses, but it can accommodate forecast error and stochastic demand. Supply-side agents are allowed to submit several blocks and to update their offer strategies based on the results of their participation in a market. The block offers are connected with each other by a fitting equation whose shape is a hockey stick. However, there is an initial value dependence. Furthermore, it is difficult to determine which parts of the data are relevant for estimating a future state. Consequently, a large and perhaps inappropriate amount of data may be required, which may result in a high computational cost and inaccuracies in any future estimates.

In this paper, a numerical optimization process is used to find a set of parameters for an optimal offer based on a double layer diffusion model developed by the authors [1]. The behavior of the agent depends on its assessment of the behavior of its competitors. According to nonlinear dynamic theory [9], a change in state dimension implies that a dynamic system has changed to either a new equilibrium or to a chaotic state. If a system undergoes change in its state dimension, the Liapunov dimension yields an estimate of how fast it approaches the new state. To identify any change in the offer strategies of competitors, this theory is applied and the simulation results are discussed.

\section{Simulation EnVironment}

Agents develop rules for themselves based on the rules of the auction they are participating in and, in repeated auctions, based on the actions of their competitors. In the design considered here, the electricity market is assumed to be based on locational marginal pricing (LMP) with both supply and demand-side participation [10]. Time-varying stochastic load demand is assumed. The network for the simulation is a system shown in Fig. 1, which is modified from the IEEE 30-bus system of reference [11]. The line capacities connecting Area 2 with rest of the system have been modified in order to generate a load 


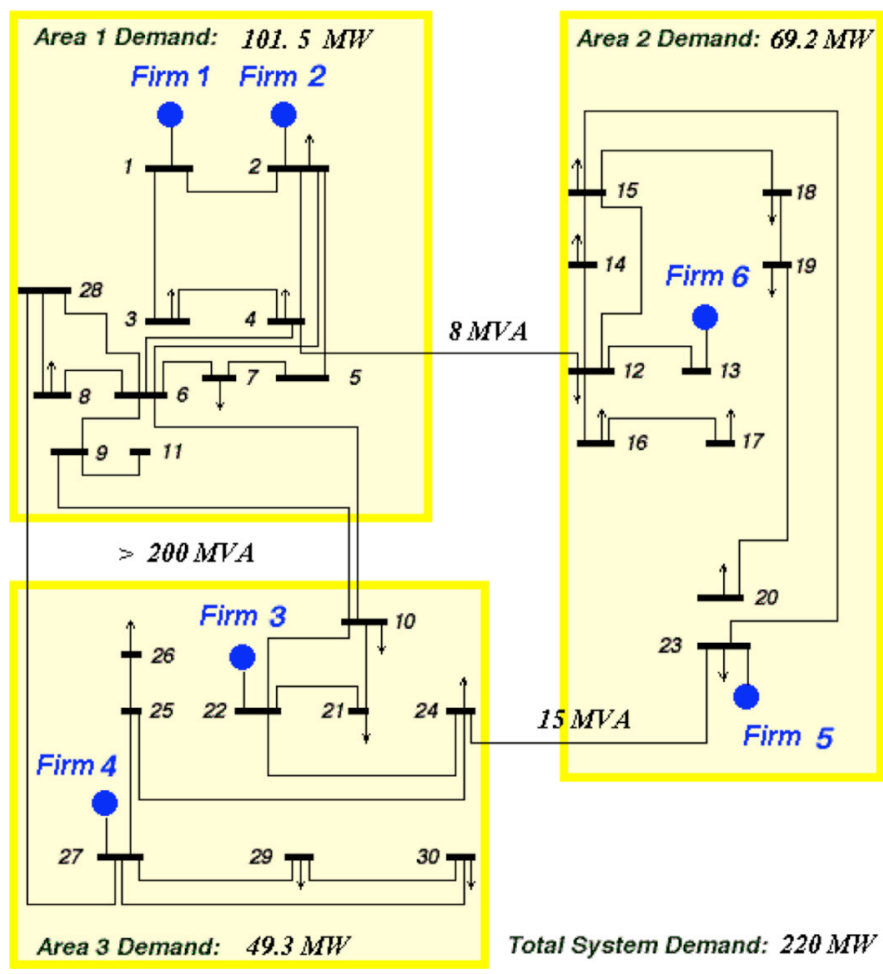

Fig. 1. Modified IEEE 30-bus system with six suppliers. The capacities of lines connecting Area 1-Area 2 and Area 3-Area 2 are lower than those of other lines.

pocket. Furthermore, the locations of some generators were also changed. In this market and for this example system, an ISO provides a load forecast and collects the offers and bids submitted by six participating agents. The ISO then clears the market and checks the security of the system.

In every period, each supply-side and demand-side agent is asked to submit a price and quantity. The ISO then runs an ac optimal power flow (OPF) to clear the market. All dispatched quantities are paid their LMPs. After the auction has been cleared by the ISO, each agent receives private information such as its dispatch quantity and price. LMPs are publicly available information.

Each supply-side agent could submit offers for as many as 30 blocks. Based on its maximization algorithms, available historical data, and load forecast, each agent decides the quantity and price it will offer. Exchange of information among agents is not allowed. However, it was assumed that a supply-side agent had access to information about the network.

\section{MARKET MODELING}

To explain experimental results performed on an unknown complex system, a linear combination is one of the most powerful and commonly used techniques in quantum mechanics. When there exists a basis set, an unknown market can be expressed in terms of the set in conjunction with the probability distribution.

It is possible to identify offer behavior in terms of a basis set. If the basis set is orthonormal, the probability of each set is simply an inner product between each set and the unknown market. However, the form of a basis set is not necessarily expressed by an offer curve. Suppose there is a convenient set in which to express offer behavior such as the weak speculators (WS), strong speculators (SS), etc. discussed in [1]. Then the elements in the set can be spanned in terms of the basis set since they exist in an offer space.

It is important to note that all the information about the network is embedded in the elements of the basis set. If the chosen set has a large enough number of elements, there exists an inverse relation that allows an inverse expansion or projection to the offer space. Consequently, one can find an expansion of an unknown offer behavior in terms of a known offer set with a weighting factor distribution. Therefore, it is possible to classify offer behavior as was shown in [1] under the mild assumptions that there are enough elements in the set and that the unknown offer is consistent. An insufficient set will result in a proper classification but with an error. The classification is not wrong as long as all the extreme offer behaviors are included in the set. Inconsistent offer behavior will lead to an overall offer behavior that could be classified properly if different behaviors are correctly analyzed.

Similarly, the state of a market is a cumulative sum of the effects of individual agents in the market. It is easier to model an individual agent than an unknown market, especially when network effects are considered. Suppose there is a market with known offer behaviors for each of its participants. Then the market can be expressed in terms of the cumulative offer behaviors of the competitors in the set, which is termed a scenario. Consequently, a market with unknown offer behavior can be expressed in terms of known offer behavior or scenarios. Then any experimental observations can be interpreted as the cumulative sum of participation in each scenario.

If a market's state does not change, then participation by competitors must have driven it parallel to its eigenvectors. In general, an orthonormal basis set is commonly selected due to the calculation convenience. For an unknown market, it is not possible to derive eigenvectors analytically or experimentally. When a market is expanded in terms of an arbitrarily set, the coefficients imply the weight factor distribution since the set is no longer a complete basis set. One can write an expression that describes market participation in terms of, for example, the earnings of an agent with given weight factor distribution c, as follows:

$$
\begin{aligned}
& {\left[\begin{array}{cccc}
\left\langle\phi_{1}|H| \psi_{1}\right\rangle & \left\langle\phi_{1}|H| \psi_{2}\right\rangle & \cdots & \left\langle\phi_{1}|H| \psi_{N}\right\rangle \\
\left\langle\phi_{2}|H| \psi_{1}\right\rangle & \left\langle\phi_{2}|H| \psi_{2}\right\rangle & \cdots & \left\langle\phi_{2}|H| \psi_{N}\right\rangle \\
\vdots & \vdots & \ddots & \vdots \\
\left\langle\phi_{m}|H| \psi_{1}\right\rangle & \left\langle\phi_{m}|H| \psi_{2}\right\rangle & \cdots & \left\langle\phi_{m}|H| \psi_{N}\right\rangle
\end{array}\right]\left(\begin{array}{c}
c_{1} \\
\vdots \\
c_{N}
\end{array}\right)} \\
& =\left(\begin{array}{c}
\left\langle\phi_{1}|H| \Psi\right\rangle \\
\left\langle\phi_{2}|H| \Psi\right\rangle \\
\vdots \\
\left\langle\phi_{m}|H| \Psi\right\rangle
\end{array}\right)
\end{aligned}
$$

where the elements in the square bracket on the left-hand side $\left\langle\phi_{x}|H| \psi_{y}\right\rangle^{1}$ represent the earning of an agent for an offer $\left\langle\phi_{x}\right|$ for a market with a specific scenario $\left|\psi_{y}\right\rangle$, i.e., a simulated earning from the known scenario. The earnings on the

${ }^{1}\left\langle\phi_{i}|H| \psi_{j}\right\rangle=\$ 4 / \mathrm{hr}$ means that offer $\phi_{\mathrm{i}}$ under rules $\psi_{\mathrm{j}}$ produced an earning of $\$ 4 / \mathrm{hr}$ where $H$ is the operator that maps offer $\phi_{\mathrm{i}}$ to earning under rules $\psi_{\mathrm{j}}$. 
right-hand side of (1) are actual earnings for the same offer submitted into an actual market $|\Psi\rangle$. When a scenario is found to be irrelevant to the expression describing the market, the scenario set can be modified without recalculating the entire matrix.

A well-defined weight factor distribution minimizes the difference between the inner product on the left-hand side and the vector on the right-hand side. Since the set is arbitrarily chosen and implemented given an uncertainty in earnings, the simulated earnings must contain errors. In this paper, the two-norm is used for the error minimization problem. The error minimization yields an optimal weight factor distribution, which allows one to model an unknown market. It is possible to evaluate earnings from a known market $\left\langle\phi_{x}|H| \psi_{y}\right\rangle$ at a given offer $\left\langle\phi_{x}\right|$. Consequently, earnings from an unknown market $\left\langle\phi_{x}|H| \Psi\right\rangle$ can be estimated by using the optimal distribution from which one can construct a numerical function mapping offers to earnings.

\section{SUPPly-SIDE AGENT}

To check for any change in the strategy of other agents, state dimension and its Liapunov exponent are calculated. The market situation is evaluated by using data obtained during a period where there is no change detected. Based on historical data of forecast and actual demand obtained under the same market situation, actual demand for the next period is estimated using a neural network. With a given estimated demand, an agent could construct a numerical mapping function from offer to earnings. Theoretically, it is also possible to find an optimal offer analytically since the weight factor distribution does not depend on the offer. However, it needs multiple function evaluations, which is computationally expensive. A trust-region method [12] has been used successfully to find an optimal solution with minimal function evaluation. Since all offer parameters have upper and lower bounds [1], it is suitable for this problem to use the trust-region method for nonlinear optimization subject to bounds.

\section{Simulation Results and Discussion}

Several simulations were performed using the agents described in the previous sections. All demand-side agents had a must-serve demand of about $10 \%$ [10]. To see the performance, only one supply-side agent was used while standardized agents described in [1] represented other firms. To emulate different situations, several markets with different distributions of competitors ranging from competitive to volatile were simulated.

The agents were tested in terms of their reaction to a permanent and consistent change in strategy by others. For the test, a competitor changed its strategy completely and can detect the change in strategy. In one simulation after 253 periods, Firm 6 changed its strategy while Firms 1-4 did not. The results for historical nodal price data obtained from competitive and volatile markets are shown in Fig. 2.

Before Firm 6 changes its strategy, the relative errors between simulated and actual earnings of the agent representing Firm 5 were about $65 \%$. Modification of the scenario set reduces the error to $59 \%$. With the new modified scenario set, the agent tried to find an optimal offer for given load forecast.

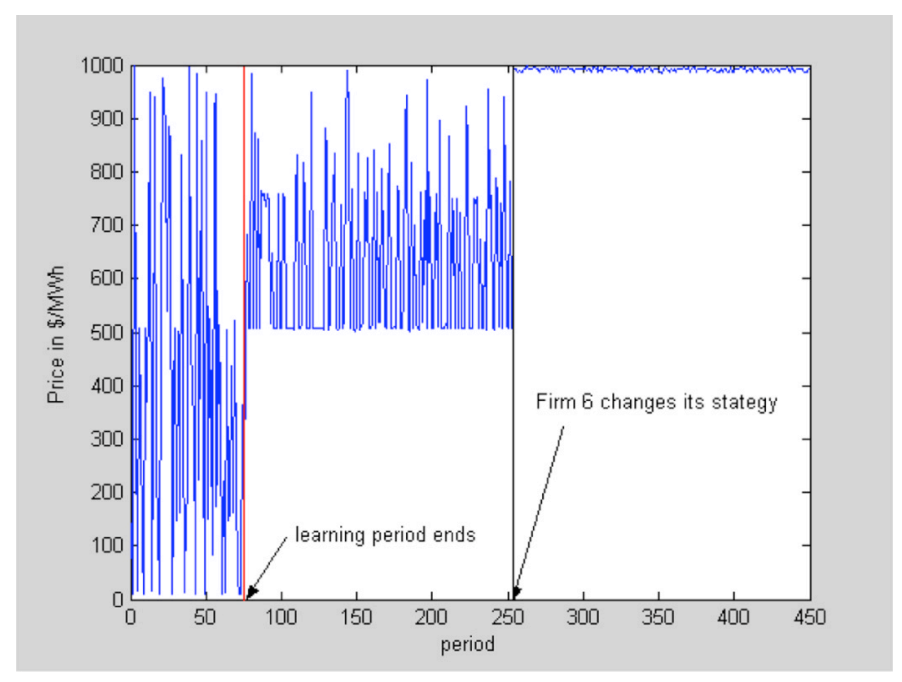

Fig. 2. Historical data for nodal prices of Firm 5 when Firm 6 changes its strategy from marginal cost offer to speculator.

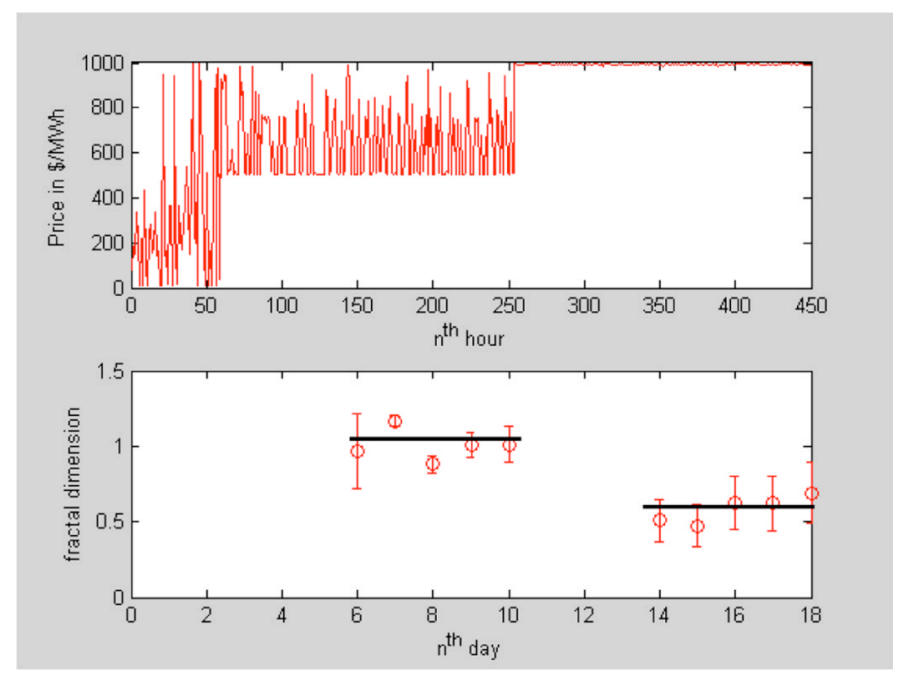

Fig. 3. Daily dimension check for the historical nodal price data obtained from the simulation described in Fig. 2.

The dimension check was performed in terms of detecting a possible change in strategies of other competitors. Fig. 3 shows that the dimension check detects a possible change in the state of market about four days after Firm 6 changes its strategy. Note that at least 100 data points are required to evaluate the dimension with a value of 2 or higher for statistical reasons. Several such simulations were run and showed that the dimension check reliably detects the changes in market state.

When the state of the market changes, it is important to find how long the change takes to settle down a new state. The question can be answered by evaluating Liapunov exponent. In general, it takes two times the inverse exponent for the system to reach equilibrium. Fig. 4 shows the results of an evaluation of the exponents. In days 12 and 16 after the change was made, the exponents take very small values while most others are negatives. In evaluating the Liapunov exponent, only a few points were randomly taken to avoid choosing biased data, and the actual value of the exponent greatly depends on the choices made 


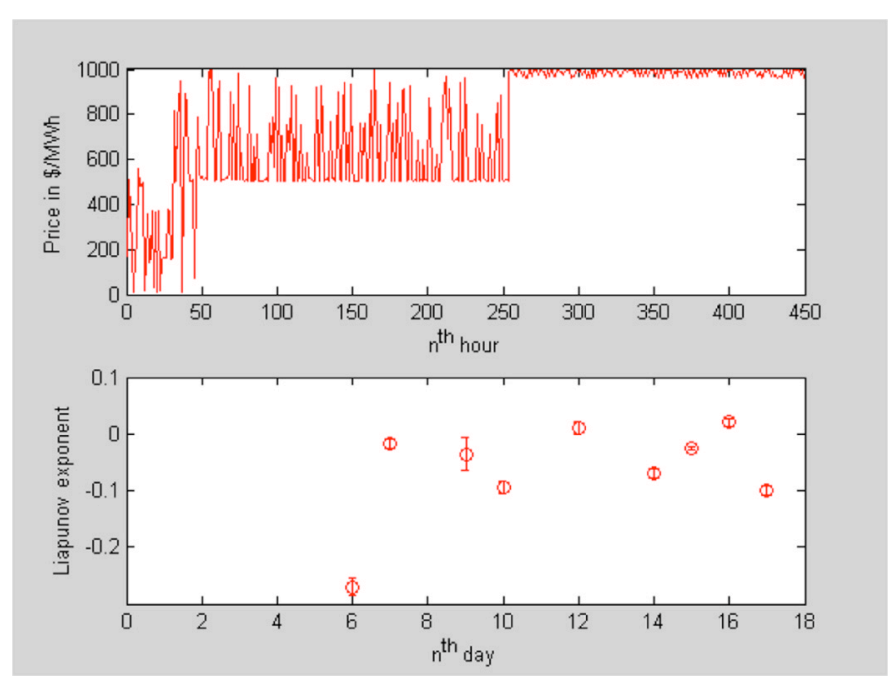

Fig. 4. Results of Liapunov exponent calculation performed with the historical nodal price data obtained from the simulations described in Fig. 2.

in selecting the points. Consequently, the value of the exponent is less reliable when it is small. For negative or zero values of the exponent, an agent chooses a default number of data for calculating weight factors. When the exponent has a large positive value with a small error, it says that the system settles down to a new equilibrium quickly. A small positive exponent value like in these cases implies that the dynamics are very slow, in which case many data points are relevant to the future estimate. Since the exponent tells how recent data are relevant to estimating a future state, it only gives an upper limit for the number of data that an agent must choose. In both cases here, more than 100 data points are at their upper limits.

From the results of the dimension check, the performance of an agent before finding the change in state seems as good as that after finding it. A dimension check and Liapunov exponent allow an agent to choose relevant data. Before a change of state, a dimension check gives no change and a Liapunov exponent calculation gives a negative value. In this case, an agent is allowed to have only 100 data points. Before the agent realizes a change in state has occurred, several new data have been added into the 100 data points. Consequently, the effect of the new addition is not negligible. Without using the dimension and exponent, the agent will carry all the data, and then the effect of adding new data is small enough to be ignored. Thus, the performance could not be comparable to an optimal one. For a randomly generated offer, the nodal price fluctuates substantially as is shown during a learning period in Fig. 2. Once the agent finds a proper weight factor distribution, the price is not below $\$ 500 / \mathrm{MWh}$.

The agent finds an optimal offer to maximize its profit with a given weight factor distribution and forecast. As was mentioned before, the agent manages to keep the nodal price no less than $\$ 500 / \mathrm{MWh}$ while the price fluctuates at random offers. Fig. 5 shows the historical and estimated earnings. The average error after learning period and before the change in the strategy of Firm 6 was $17 \%$ and that after the change was $1 \%$. A competitive market requires an agent to explore offer-earning surface

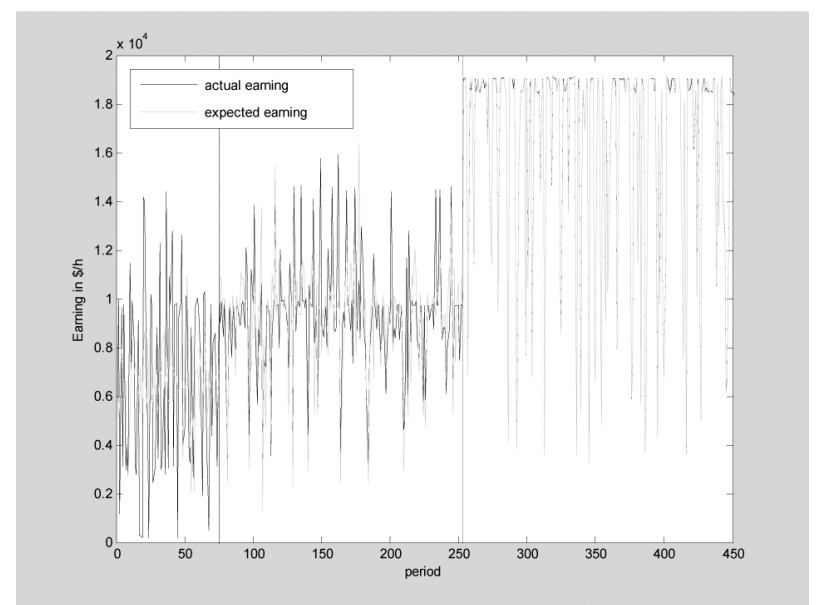

Fig. 5. Historical data for the actual and expected earning of Firm 5 in black and gray lines, respectively.

widely, which needs more basis set. With the given number of the basis set, the error would be larger in a more competitive market than that in a less competitive one.

To study an offer strategy, the degree of speculation ${ }^{2}$ and the maximum offer price ${ }^{3}$ described in [1] from the learning period and periods before and after the sudden change are plotted in Fig. 6(a). Both graphs in the first row of Figs. 6(a) show the variables chosen for offers during the learning period. The graphs in the second and third rows illustrate the variables before and after the sudden changes. The second row corresponds to the simulation against more competitive agents. The variables from the simulations against more competitive agents seem to fluctuate more often, but it is difficult to find any difference in the offer strategies between before and after the change.

The ratio between the two variables was calculated and plotted in Fig. 6(b). The data clearly show that the ratio increases when the market becomes less competitive.

When the market is less competitive, the agent can take advantage of the other agents by being less speculative. This results in reducing the value of the degree of speculation and/or offering more quantity into the market. Both cases increase the ratio by decreasing the denominator and by increasing the numerator from the former and latter case, respectively. Note that the agent would increase its earning in a competitive market by being more speculative when the agent has locational benefit.

For testing the performance for the case where the agent does not have a locational benefit, a simulation was performed with an agent participating on behalf of Firm 1 in the same situation described before. The results are shown in Fig. 7.

Similar to the previous case, the changes in the price at Bus 1 are approximately identical to those in the case described in Fig. 2. The height and the frequency of the price spikes are a little lower than before. Before Firm 6 changes its strategy, the relative error was about $58 \%$. For a better estimate, the agent modifies the scenario set modeling its neighboring competitors

\footnotetext{
${ }^{2}$ The distance from the fair share to the quantity that deviates from a low price offer

${ }^{3}$ The highest price appeared on an offer curve
} 


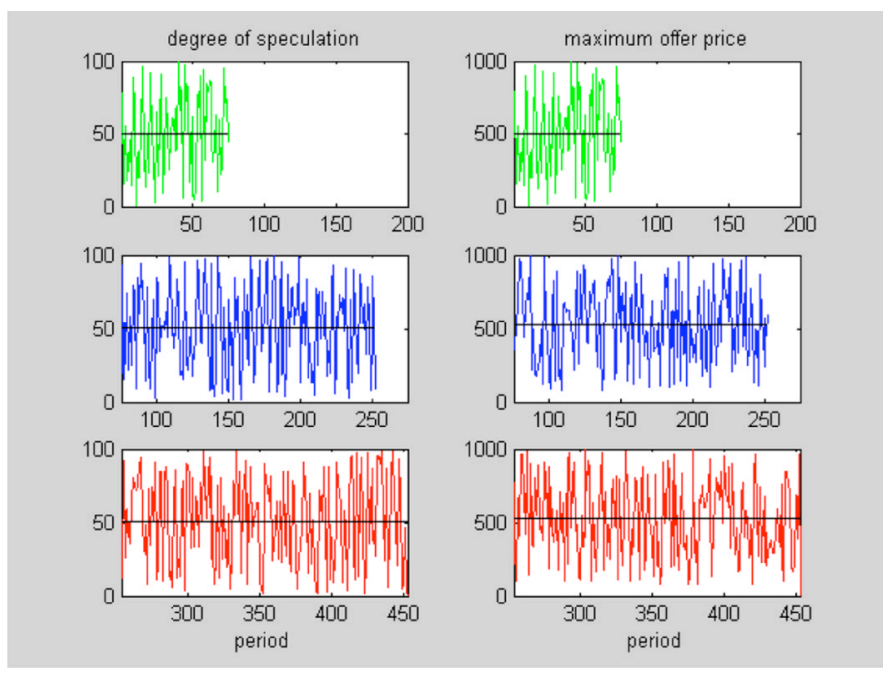

(a)

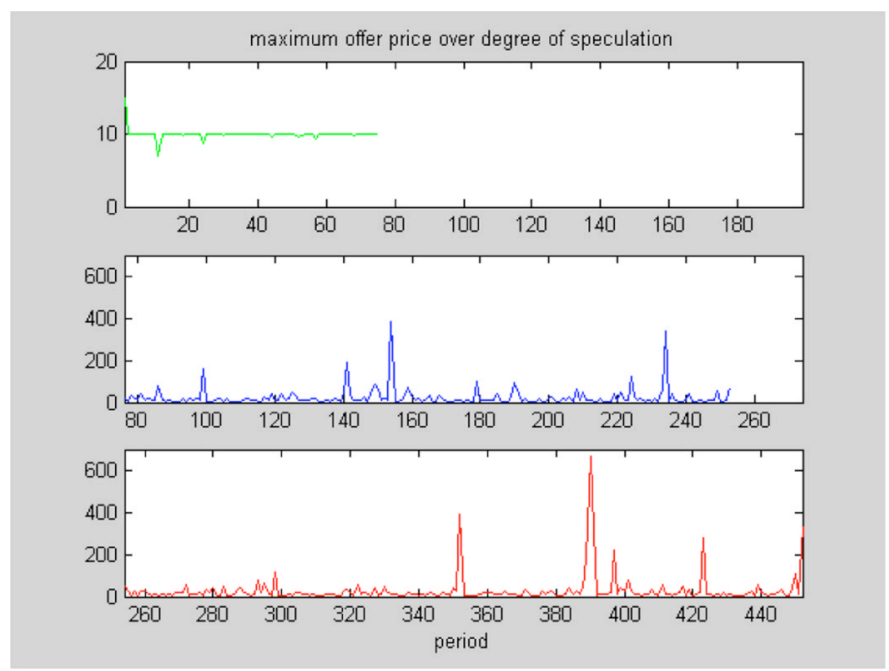

(b)

Fig. 6. (a) Degree of speculation and the maximum offer price changes over periods obtained; horizontal lines indicate the mean values of the variables within the period shown on the horizontal axis. (b) Ratio of the maximum offer price to the degree of speculation over the periods shown on the horizontal axis; the corresponding values of both variables are shown in Fig. 6(a).

Firm 2, 3, and 4 while leaving scenarios for Firm 5 and 6 unchanged. Modification of the scenario set described above makes error reduced to $51 \%$. With the new modified scenario set, the agent tried to find an optimal offer at given load forecast.

A daily dimension check was also performed to check a possible change in strategies of other competitors. Fig. 8(a) shows that it detects a conceivable change in the state of market in a couple of days after Firm 6 changes its strategy. For the changed market, the agent evaluates the Liapunov exponent in terms of detecting the duration of the change to settle into a new state. Fig. 8(b) shows the results of evaluation to the exponents. No exponents before and after the change have sufficiently large positive values. The results imply that there was a consistent change

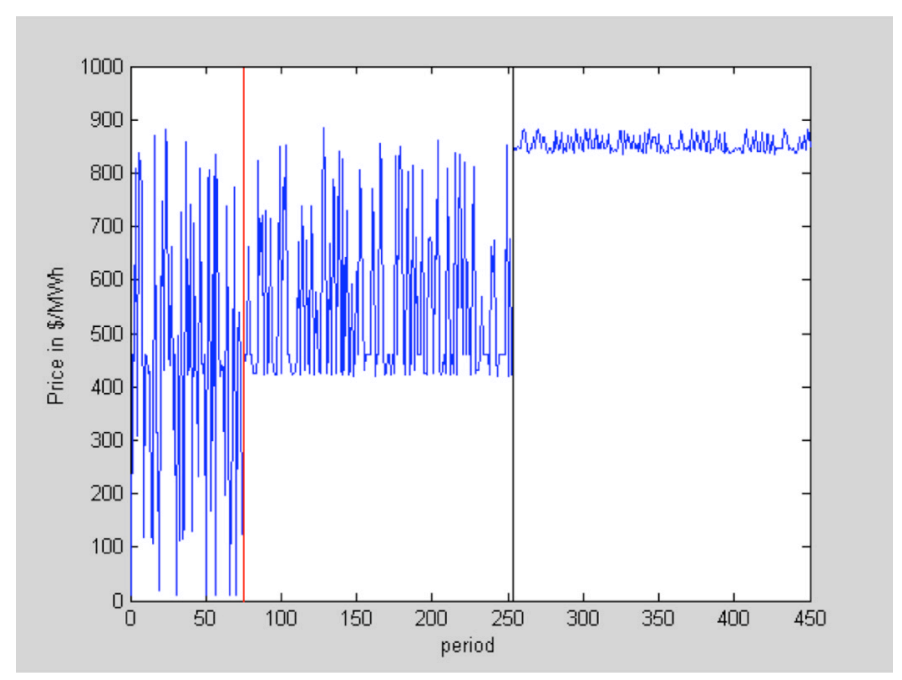

Fig. 7. Historical data for nodal prices at the Bus 1, which has no locational benefit; Firm 6 changes its strategy in a more speculative way completely at the period of 253 .
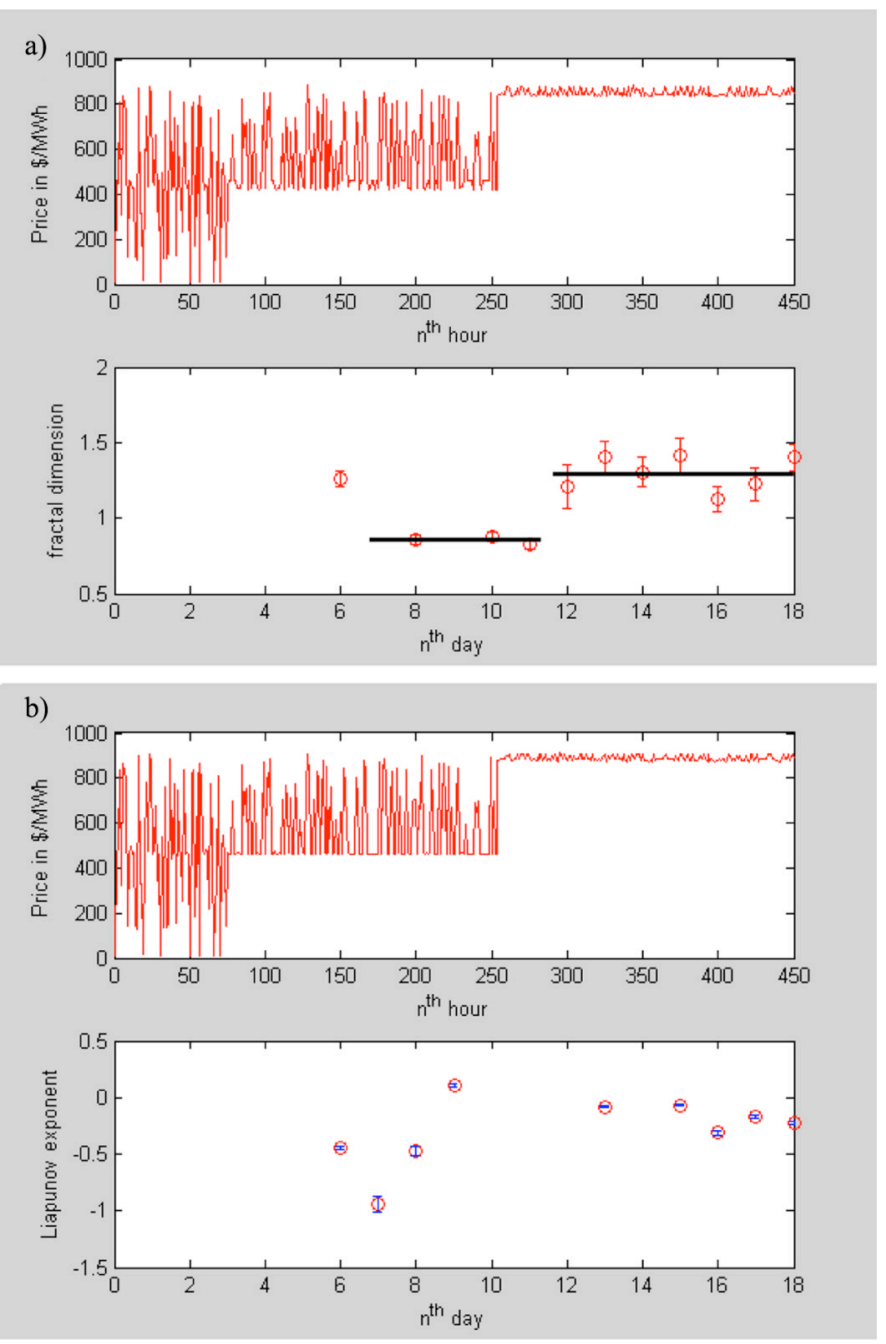

Fig. 8. Daily check for a) dimension and b) Liapunov exponent calculated with the historical nodal price data obtained from the simulations. 


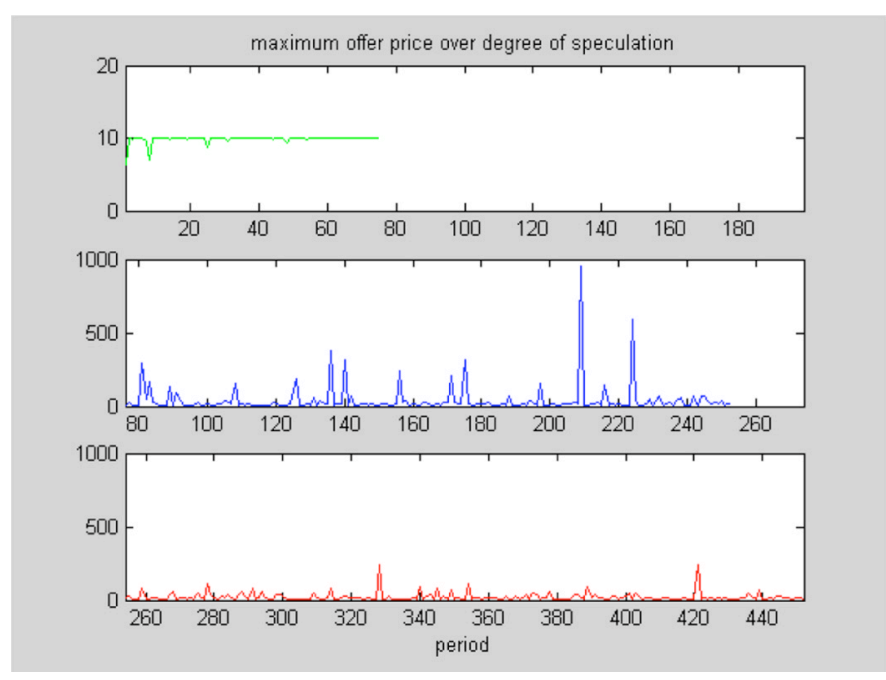

Fig. 9. Ratio between the variables over several periods.

in the strategies of its competitors, and the market settled into a new state quickly. At Day 9, the Liapunov exponent shows a positive value, but the dimension check implies no change in the state. In such a case, the Liapunov exponent is ignored since the dimension is more reliable.

For showing the offer behavior of the agent, the ratio between the two variables was calculated and plotted in Fig. 9. The figures clearly show that the ratio decreases when Firm 6 becomes more speculative, i.e., the market that the agent faces is less competitive. It is an interesting result since the ratio changes in the opposite direction when the agent has a locational advantage. When the market is less competitive, the agent can take advantage of the other agents. However, the market does not become sufficiently volatile due to the change in the strategy of Firm 6. Consequently, the agent may have options; it can make the market more volatile by submitting more speculative offers, or it can take a smaller advantage out of the change. In the environment of the simulation, the process finds that being more speculative is better. In terms of cooperating with the firm that changed its strategy, the agent tries to help by withholding more or submitting more speculative offers.

The former decreases the maximum offer price while the latter increases the degree of speculation. Consequently, the ratio between two variables decreases when the competitor changes the strategy to a more speculative way. For an agent without locational benefit, its generating unit may not be required to meet the demand. In such a case, speculation may increase the probability that its unit does not get dispatched. In order to avoid the situation, it tries to compete against other firms. Then it may decrease the degree of speculation or withhold less quantity. Therefore, the ratio increases, which is the opposite case when the agent has locational benefit.

The agent manages to keep the nodal price about $\$ 500 / \mathrm{MWh}$ and $\$ 900 / \mathrm{MWh}$ before and after the change in the strategy of Firm 6, respectively. Fig. 10 shows the historical and estimated earnings from the simulation. The error after the learning period and before the change in the strategy of Firm 6 was 19\%, and that after the change was $1 \%$.

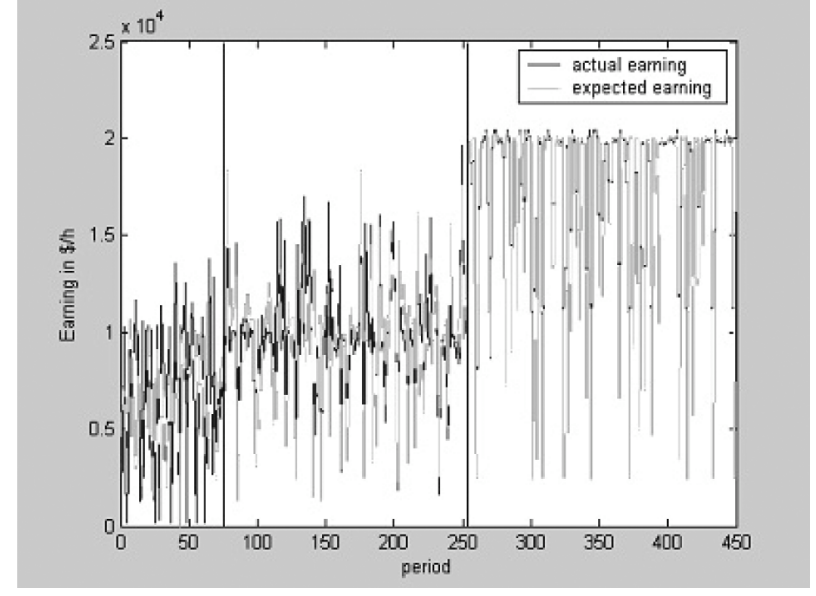

Fig. 10. Historical data for the actual and expected earning of Firm 1 in black and gray lines, respectively.

\section{CONCLUSIONS}

Agent-based simulations provide insights into strategic behavior in a large nonlinear system like the electricity power industry. With the double layer diffusion model, an offer curve can be constructed by using several parameters. Modeling an unknown market along with the parameters allows to construct a numerical function mapping from offer to earning.

Nonlinear dynamic theory can be used to identify a change in behavior successfully by using available data. The ratio between the parameters determining offer curve is an important parameter to understand the offer strategy of an agent in a given condition. The locational benefit plays a critical role in determining the optimal offer strategy.

\section{REFERENCES}

[1] H. Oh, R. J. Thomas, B. Leiseutre, and T. Mount, "A method for classifying offer strategies observed in an electricity market," J. Decision Support Syst., vol. I, Special Issue on Competitive Electricity Market, pp. 449-460, 2005.

[2] C. Richter and G. Sheblè, "Genetic algorithm evolution of utility bidding strategies for the competitive marketplace," IEEE Trans. Power Syst., vol. 13, no. 1, pp. 256-261, Feb. 1998.

[3] C. Richter, G. Sheblé, and D. Ashlock, "Comprehensive bidding strategies with genetic programming/finite state automata," IEEE Trans. Power Syst., vol. 14, no. 4, pp. 1207-1212, Nov. 1999.

[4] C. Ritcher and G. Sheblè, "A profit-based unit commitment GA for the competitive environment," IEEE Trans. Power Syst., vol. 15, no. 2, pp. 715-721, May 2000.

[5] D. W. Bunn and F. S. Oliveira, "Agent-based simulation-an application to the new electricity trading arrangements of England and Wales," IEEE Trans. Evol. Comput., vol. 5, no. 5, pp. 493-503, Oct. 2001.

[6] D. W. Bunn, "Forecasting loads and prices in competitive power markets," Proc. IEEE, vol. 88, no. 2, pp. 163-169, Feb. 2000.

[7] _ "Evaluation of market power using agent based simulation," in Proc. IEEE Power Eng. Soc. General Meeting, 2003, vol. 1, pp. 506-508.

[8] T. Mount and H. Oh, "On the first price spike in summer," in Proc. 37th HICSS Conf. System Science, 2004. [Online]. Available: http://csdl.computer.org/comp/proceedings/hicss/2004/2056/02/ 205620050a.pdf.

[9] S. H. Strogatz, Nonlinear Dynamics and Chaos. Cambridge, U.K.: Westerview, 2000.

[10] H. Oh and R. J. Thomas, "An agent-based optimal bidding function," in Proc. 39th HICSS Conf. Syst. Sci., 2006. [Online]. Available: http://csdl2.computer.org/comp/proceedings/hicss/2006/2507/10/ 2507100242c.pdf. 
[11] O. Alsac and B. Stott, "Optimal load flow with steady state security," IEEE Trans. Power App. Syst., vol. PAS-93, no. 3, pp. 745-751, 1974.

[12] T. F. Coleman and Y. Li, "On the convergence of reflective newton methods for large-scale nonlinear minimization subject to bounds," Math. Program., vol. 67, pp. 189-224, 1994.

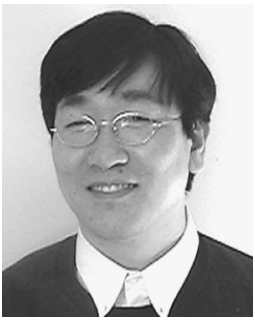

HyungSeon Oh received the MS. degree in materials science and engineering and the Ph.D. degree in electrical and computer engineering from Cornell University, Ithaca, NY, in 2002 and 2005, respectively.

He is currently a Postdoctoral Research Associate with the Department of Electrical and Computer Engineering, Cornell University. His research interests includes stochastic optimization, electric power economics, computer simulation, and nonlinear dynamics.

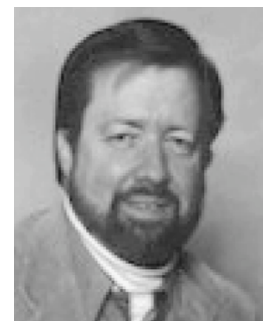

Robert J. Thomas (F'93) is a Professor of electrical and computer engineering at Cornell University, Ithaca, NY. His current research interests are broadly in the areas of analysis and control of nonlinear continuous and discrete-time systems with applications to large-scale electric-utility systems.

Dr. Thomas has been a member of the IEEE United States Activity Board's Energy Policy Committee since 1991 and was the committee's Chair from 1997 to 1998. He was a member of the IEEE Technology Policy Council, has served as the IEEE-USA Vice President for Technology Policy, and has been a member of several university, government, and industry advisory boards or panels. 